zustand der Pollen in dieser Schicht spricht jedoch andererseits eher für eine genügende Wasserbedeckung während der Sedimentation.

Literaturverzeichnis

Jessen, K. \& Milthers, V.: Stratigraphical and Paleontological Studies of Interglacial Freshwater Deposits in Jutland and Northwest Germany. - Danmarks Geol. Unders. II. Raekke No. 48, Kobenhavn 1928.

KeILHACK, K.: Erläuterungen zur Geologischen Karte von Preußen. Blatt Lüneburg. Berlin 1922.

MüLleR, G \& Weber, C. A.: Über eine frühdiluviale und vorglaziale Flora bei Lüneburg. - Abh. preuß. geol. Landesanst. N. F. 40, Berlin 1904.

Thomson, P. W.: Das Interglazial von Wallensen im Hils. - Eiszeitalter und Gegenwart 1, Öhringen/Württ. 1951.

Woldstedt, P.: Uber die stratigraphische Stellung einiger wichtiger Interglazialbildungen im Randgebiet der nordeuropäischen Vereisung. - Z. deutsch. geol. Ges. 99. 1949 - Norddeutschland und angrenzende Gebiete im Eiszeitalter. K. F. Koehler-Verlag, Stuttgart 1950.

Woldstedt, P., Rein, U. \& SELle; W.: Untersuchungen an nordwestdeutschen Interglazialen. - Eiszeitalter und Gegenwart 1, Öhringen/Württ. 1951.

Ms. eingeg.: 19. 1. 52

\title{
Paléoclimats Quaternaires et Morphologie Climatique dans le Midi Méditerranéen
}

\author{
par J. Tric a r t
}

Directeur du Laboratoire de Géographie de l'Université de Strasbourg

Les géomorphologues français découvrent sans cesse de nouvelles traces des actions périglaciaires quaternaires, comme cela a été reconnu depuis longtemps en Allemagne et aux Pays-Bas. Ces dernières s'avèrent avoir eu une importance primordiale sur la genèse du relief. La plus grande partie du modelé que nous avons sous les yeux est figée et se compose de formes mortes, d'âge périglaciaire dans la plupart des cas, à peine retouchées par une médiocre érosion postglaciaire s'exerçant au surplus dequis quelques millénaires seulement.

Jusqu'à ces derniers temps, les spécialistes pensaient généralement que le Bassin Méditerranéen était resté à l'écart des influences périglaciaires quaternaires et n'avait connu que des alternances de climats plus secs et plus humides (1). Mais Guilcher (2) a signalé des phénomènes dus au froid dans la région de Lisbonne. Dans deux articles tout récents, parus après nos propres observations sur le terrain, Baeckeroot (3) et Marcelin (4) ont décrit des faits analogues, l'un aux altitudes moyennes de la Montagne Noire (niches de gélivation, pinacles, éboulis ordonnés etc. . vers $700 \mathrm{~m}$. d'altitude), l'autre dans da Garrigue Nîmoise. La limite méridionale en France des actions périglaciaires quaternaires est donc remise en question.

Or, étant donné l'importance primordiale des actions morphogénètiques du froid, ces découvertes sont susceptibles de modifier profondément l'explication du relief de vastes régions de la France Méditerranéenne et des pays voisins (Catalogne, Ligurie etc...). Nous voudrions ici, à la suite d'un voyage de recherches de trois semaines dans le Languedoc et la Provence Occidentale, montrer quelques uns des aspects de ce problème afin de faciliter les recherches futures en les orientant vers les points les plus importants. 


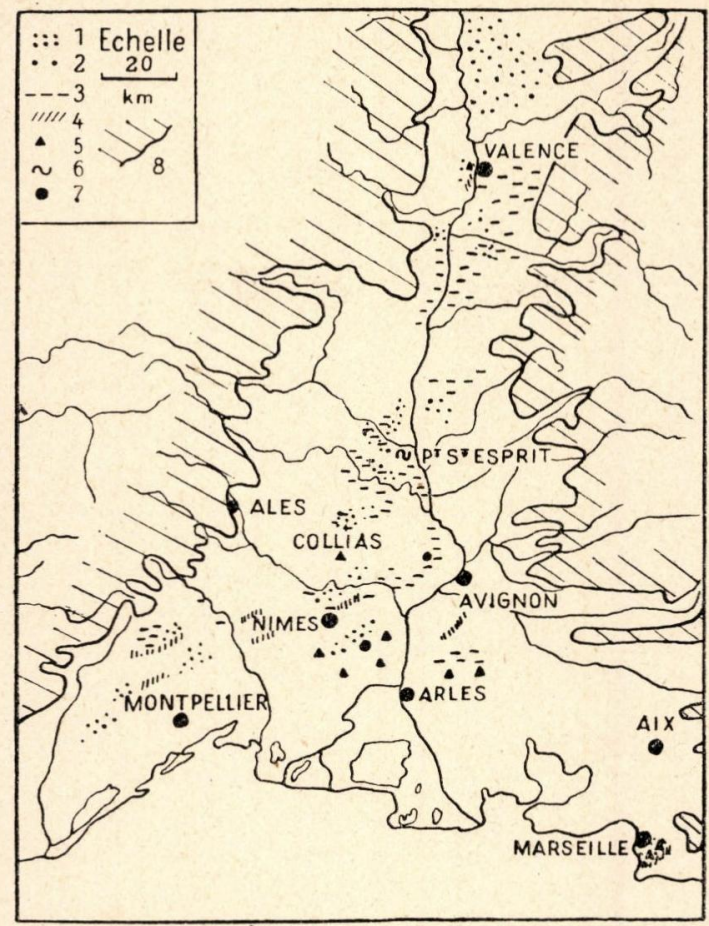

FIGURE 1: Localisation de quelques dépôts et phénomènes périglaciaires dans le Sillon Rhodanien et le Midi Méditerranéen.

1 Loess (épais)

2 Sable éolien, cailloux éolisés

3 Grèves calcaires

4 Eboulis ordonnés
5 Coulées de solifluction amorphes

6 Cryoturbation (injections, Brodelböden)

7 Grandes villes

8 Régions montagneuses (au dessus de $800 \mathrm{~m}$ ).

I Les Paléoclimats d'aprés les Paleosols.

Dequis longtemps déja, Marcelin avait signalé, dans l'E. du Languedoc, des loess et des sols loessiques (13) et insisté sur l'importance des paléoclimats pour expliquer la pédologie de cette région. Les magnifiques galets éolisés du BasRhône avaient également été décrits par de nombreux auteurs (voir 4 et la bibliographie qui y est contenue.) Carlleux, à la suite de la découverte de faits analogues dans le Roussillon, a été conduit à poser le poblème de la limite des éolisations quaternaires dans le monde méditerrannéen (6). Mais jusqu'à présent, il n'a pas été tiré de conclusions paléoclimatiques certaines de tous ces faits. La découverte, essentielle, des loess languedociens par MARCELIN a été prématurée, en ce sens qu'elle a été faite à une époque oú l'origine de ces formations était encore mal connue et qu'il état difficile de les interpréter.

A l'heure actuelle, l'origine éolienne des loess est admise à peu près par tous (7) de même que leur relation avec les climats froids et secs des périodes glaciaires. En France du Nord, en Alsace, en Allemagne Moyenne, il semble que les loess qui ont réussi à se conserver jusqu'à nos jours, se soient déposés lors de la fin des périodes périglaciaires, au moment oú les phénomènes de solifluction et de cryoturbation commençaient de s'atténuer (8). L'existence de loess typique permet donc d'inférer l'existence d'un climat de type périglaciaire.

De la fréquence des loess dans la région Nîmoise, reconnue par Marcelin, nous pouvons conclure à l'importance des phénomènes périglaciaires quaternaires. 
Mais tous ces loess ne sont pas contemporains comme l'avait bien pressenti Suen (19). Ils appartiennent à des périodes froides successives, séparées par des oscillations plus chaudes, au moins autant que le climat actuel. C'est ce que montre l'étude d'une coupe déjà bien connue, signalée autrefois par Marcelin, celle de Collias (Gard).

Dans la tranchée, profonde de 8 à $10 \mathrm{~m}$., de la route qui mène de ce village à Sanilhac, nous avons relevé la succession suivante (début mars 1951):

I première série, discordante sur la seconde qu'elle recoupe obliquement et qu'elle ravine.

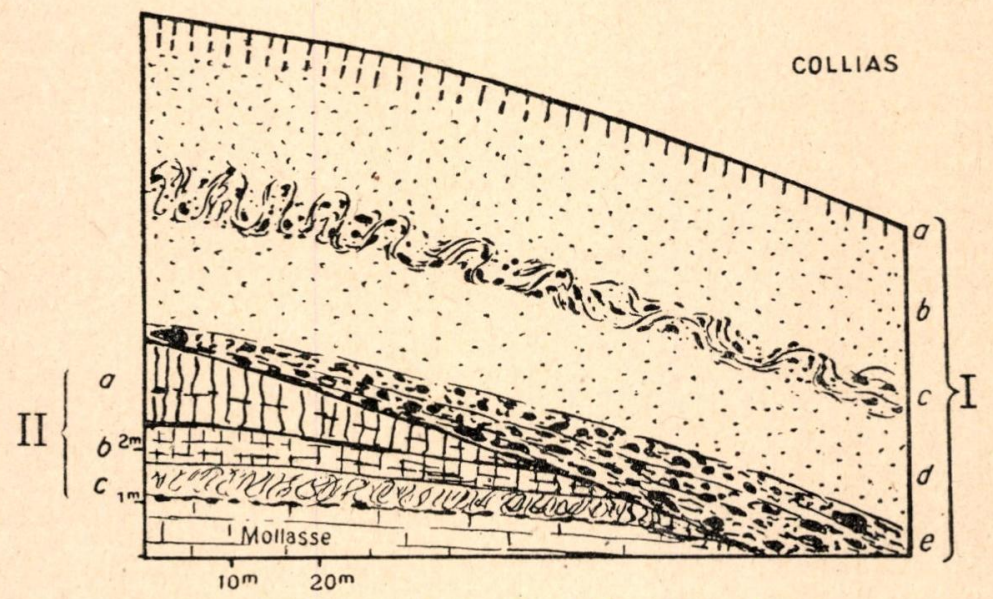

FIGURE 2: Coupe de Collias (Gard).

a) Terre végétale, constituée par un loess très légèrement lehmifié, panaché, à points noirs, sans niveau de poupées à sa base, passant graduellement à b) $0,2-0,4 \mathrm{~m}$.

b) Loess typique à fines tubulures calcaires semblant dues à des mychorizes de gazon, et à points blancs

$3 \mathrm{~m}$.

c) Couche cryoturbée, en forme de volutes et de poches, du genre Brodelboden, plus fortement derangée à sa partie inférieure.

Le sommet est formé d'un loess brassé avec des éléments de lehm et des poupées, mélangés à quelques petits graviers calcaires (moins de $1 \mathrm{~cm}$ ). 0,75 m. La base, constituant un Brodelboden typique, brasse des poches de sable légèrement éolisé (Flugsand) et de loess lehmifié. Des poupées impures et diffuses se sont constituées postérieurement au contact du sable et du loess lehmifié. $0,0-0,5 \mathrm{~m}$.

d) Loess doux avec rares poupées à son sommet et panachures blanches. Non cryoturbé $2 \mathrm{~m}$.

e) Lit de graviers anguleux, du type produits de gélivation, $(\mathrm{L}=1-6 \mathrm{~cm})$, ravinant la couche sousjacente à laquelle ils empruntent leur matrice. Celleci est parfois consolidée. Vers le bas du versant, ces couches se multiplient et s'épaississent en même temps que leurs éléments deviennent plus grossiers (blocs atteignant $30 \mathrm{~cm}$ ). Elles ravinent alors directement la molasse altérée $0,10-1,5 \mathrm{~m}$.

I I d e u xi ème sé ri e, ravinée par la première et conservée seulement au point culminant de la route, entaillant une petite croupe. De chaque côté, elle a 
disparu et l'ensemble I ravine alors directement la molasse, ce qui prouve que les vallons se sont creusés entre le dépôt de cet ensemble et celui de la couche le.

a) Lehm panaché orange et rouge, très graveleux, contenant de nombreuses poupées à texture granuleuse, très différentes de celles du lehm alsacien actuel. Par endroits, ce lehm passe à sa base à un lehm argileux rouge vif, se débitant en colonettes typiques, dont la puissance maxima est de $30 \mathrm{~cm}$.

$0,20-1 \mathrm{~m}$.

b) Lehm panaché rouge et blanc, à abondantes poupées graveleuses et concrétions calcaires diffuses esquissant des lits $\quad 0,50 \mathrm{~m}$.

c) Sol d'altération orange à points blancs et noirs et lits de concrétions calcaires diffuses passant à sa base à la molasse

$1 \mathrm{~m}$.

Cette coupe remarquablement complète mérite de devenir aussi classique que celles d'Achenheim et de Hangenbieten en Alsace, car elle peut servir de point de départ à toute une chronologie pédologique du Quaternaire régional. Elle appelle les commentaires et interprétations suivantes:

$1^{\circ}$ Le sol actuel n'est pas un lehm typique: la zone de concrétionnement y est peu développée, ce qui le différencie nettement des lehms alsaciens, au surcroît plus épais, plus argileux et plus humiques. Il est vraisembiable que cette moindre altération du loess soit due aux caractères du climat méditerrannéen, dont les pluies violentes, les longues périodes de sécheresse sont moins favorables que l'humidité prolongée du climat alsacien aux phénomènes de migration et d'hydrolyse. En tous cas, ce sol suffit à montrer que le loess n'est plus en équilibre avec le climat actuel et qu'il s'altère sous son influence. Cela constitue une preuve supplémentaire de son dépôt sous un autre milieu climatique.

$2^{\circ}$ Lorsqu'ils ne sont pas altérés, les loess visibles dans cette coupe ont des faciès parfaitement typiques, ce qui implique que leurs conditions de genèse n'étaient pas sensiblement différentes de celles des loess alsaciens, donc que le climat des périodes froides à loess du Languedoc était presque le même que celui qui régnait en Alsace lors du depôt des mêmes formations.

$3^{\circ}$ Il existe, dans cette coupe, 2 loess parfaitement typiques dont une partie n'est pas altérée (couches Ib et Id), séparés par un lehm et une cryoturbation. La cryoturbation intermédiaire a affecté essentiellement la couche supérieure lehmifiée du loess le plus ancien (couche Ic). Les petits graviers calcaires dispersés dans sa partie supérieure ne forment pas des coûlées de solifluction et ils peuvent représenter des apports éoliens sur verglas (9). Dans la deuxième série de couches, on n'observe pas de loess typique mais il en subsiste les traces sous la forme d'un lehm (couches II a et b).

$4^{\circ}$ Les loess alternent avec des formations de solifluction et de cryoturbation: entre les deux loess typiques une couche cryoturbée (Ic), à la base du loess typique le plus bas, les coulées de pierraille, qui moulent une topographie entaillée dans les formations de l'ensemble II (couche Ie). Enfin, le lehm orange et rouge est également cryoturbé.

$5^{\circ}$ Les altérations des loess ne sont pas les mêmes sur toute la hauteur de la coupe. L'altération du loess typique le plus inférieur (couche Id), remaniée par cryoturbation, ne s'est accompagnée d'aucune modification des oxydes de fer, exactement comme l'altération postglaciaire (couche Ia). Il y a eu seulement migration du calcaire, ce qui semble indiquer un climat peu différent de l'actuel. Par contre les couches anciennes (ensemble II) sont profondément modifiés; les oxydes de fer ont subi une évolution hématitique qui ne se produit plus de nos jours. Ils sont à rapprocher des sols rouges. La migration du calcaire 
$\mathrm{y}$ affecte une forme particulière: au lieu de ce concentrer en concrétions bien individualisées, il se présente de manière diffuse, mêlé à l'argile et tend à s'organiser en lits. Ce ne sont plus des poupées mais presque une croûte du genre »caliche«, presque un »taparas" de Mr. Marcelin. On ne peut donc admettre pour cette altération un climat semblable à l'actuel: une température plus élevée, unę sécheresse plus accentuée furent nécessaires. Les mêmes observations sont valables pour l'altération de la molasse sous-jacente.

Il nous semble donc vraisemblable d'après cette coupe de reconstituer la succession paléoclimatologique suivante:

Période actuelle:

Période froide: dépôt de loess lors de sa fin: couche Ia couche Ib succédant à des actions éoliennes plus intenses (Flugsand cryoturbé) se produisant lors du maximum d'intensité des phénomènes périglaciaires (couche cryoturbée): couche Ic

Pé ri ode te m pér é e : de climat comparable à l'actuelle. Formation d'un lehm sur le loess de la période froide précédente, lehm qui est ensuite cryoturbé et en bonne partie détruit. Remanie la couche Ic.

Période froide: dépôt du loess comme précédemment vers la fin de la période: couche Id succédant à une phase de solifluction intense, avec formation de coulées de pierrailles:

couche Ie

Période chaude et s èche: altération d'un loess en un lehm orangé avec tendance à la formation d'une caliche: couche IIa-b

Période froide ancienne: loess entièrement altéré qui a donné naissance au lehm précédent.

$\mathrm{P}$ é ri od e $\mathrm{ch}$ a u d e e $\mathrm{t} \mathrm{s}$ è $\mathrm{ch}$ e : altération de la molasse. Climat probablement analogue à celui de la période chaude suivante: couche IIc

Il n'est naturellement pas question, en l'absence d'autres critères, de dater ces différentes phases, encore moins de les synchroniser avec les glaciations alpines. Mais on peut néanmoins remarquer une analogie qui n'est peut-être pas fortuite avec l'évolution du Bassin de Paris (10). Dans cette dernière région, le Quaternaire est caractérisé par une première période froide, relativement peu intense (11), qui succède presque immédiatement aux climats chauds du Pliocène terminal. Ensuite vient une longue phase de creusement des vallées dont le début au moins a connu la formation de croútes du genre caliche, qui cimentent les coulées de solifluction de la période froide précédente. Une nouvelle phase froide, très intense, lui succède, puis une courte période tempérée pendant laquelle l'activité morphologique a été presque négligeable, et, enfin, vient la dernière poussée de froid.

Le schéma général se retrouve ici: une première phase froide encadrée par deux périodes de climat chaud et sec (sol d'altération de la molasse, lehms rouge et orangé), et qui ne semble pas s'être accompagnée d'intense solifluction (aucune coulée de pierrailles dans ces formations rougeâtres). Une période de creusement lui succède, qui provoque la disposition discordante de l'ensemble de couches I sur l'ensemble II. Viennent alors, coup sur coup, deux périodes froides qui ne sont séparées par aucun creusement notable ni aucune altération poussée et différente de l'actuelle. La plus ancienne de ces deux dernières phases froides commence par une intense solifluction tandis que les phénomènes éoliens dominent lors de la seconde (Flugsand). Ces similitudes sont trop nombreuses pour être fortuites, mais il nous est impossible de nous prononcer sur leur signifi- 
cation. On peut cependant noter que les sols rouges sont anciens et remonteraient au Quaternaire Inférieur, ce qui s'accorde parfaitement avec les récentes découvertes des pédologues. Les sols méditerrannéens français actuels sont des sols bruns. Des rapprochements sont possibles entre la succession pédo-climatologique que nous avons observée et les régions voisines, en particulier le BasDauphiné.

Bourdier a noté que seules les plus anciennes des moraines pré-alpines portent des sols d'altération rouges ce que nous avons également observé sur les terrasses de la Plaine de Valence (environs de Malissard). Il considère ces altérations comme caractéristiques de la période interglaciaire Mindel-Riss. Si nos deux loess les plus récents (Ib et Id) représentent, comme c'est vraisemblable, les deux dernières phases froides, l'accord est complet: les sols rouges auraient cessé de se former avant le Riss et caractériseraient, outre le Tertiaire supérieur, le Quaternaire ancien. Il serait essentiel de poursuivre les recherches sur cette question, car elles sont d'une importance primordiale pour l'étude de l'évolution du relief: les nombreuses pentes tapissées de sols rouges, de la France Méridionale seraient des reliefs anciens peu ou pas modifiés au cours du Quaternaire Moyen et Supérieur. La méthode peut se révéler efficace pour la datation des terrasses.

D'autres traces d'une période froide ancienne se retrouvent dans le Sillon Rhodanien moyen. C'est, en particulier, le cas du beau gisement de St. Vallier, découvert par F. Bourdier et étudié par VIRET (20), dont la faune villafranchienne gisait, après avoir subi un faible transport, sous un loess durci, à plus de $100 \mathrm{~m}$. au dessus du Rhône. Nous avons retrouvé, en compagnie de H. Poser, de A. Cailleux et de A. Journaux, sous la direction de J. Michaud, au S. de St. Peray, dans une ancienne vallée du Rhône à l'O. de la plaine de Valence un loess durci identique, signalé autrefois par Suen (19) mais qui n'en avait donné ni interprétation ni coupe précise. Cette dépression est encombrée de formations alluviales riches en quartzites alpins et dont la morphoscopie semble fluvioglaciaire. Sur son flanc E., descendant de la colline de Crussol, s'observent sur plus de $3 \mathrm{~m}$. d'épaisseur, des alternances de loess durci et de pierraille calcaire de gélivation cimentée en brèche, qui semblent venir se raccorder aux alluvions. Non loin de là, à St. Péray, nous avons observé la superposition de deux loess dont le plus bas porte un lehm orangé (12).

Quelquesoit leur âge précis, toutes ces formations indiquent:

- l'existence de périodes froides quaternaires dans la France Méridionale, avec phénomènes du type périglaciaire,

- la succession de plusieurs alternances de climats froids et de climats chauds au Quaternaire ancien, tempérés au Quaternaire Moyen et Supérieur.

Examinons donc quelles sont les conséquences de ces oscillations climatiques sur la génèse des formations détritiques du Midi Méditerranéen.

\section{Les phénomènes périglaciaires}

C'est à Marcelin que revient l'honneur d'avoir publié la premiére liste des phénomènes périglaciaires relevés dans le région de Nîmes (4). Nous nous appuierons sur elle tout en la complétant par nos propres observations, qui portent sur une région plus étendue. Nous verrons ensuite quelles précisions paléoclimatiques il est possible de tirer des divers faciès rencontrés.

a) Les phénomènes éoliens.

Ils sont particulièrement fréquents et intenses. On peut en distinguer trois formes: 
$1^{\circ}$ Les dépôts de loess, patiemment étudiés par Marcelin. Leur importance est considérable. Des loess ou des sols loessiques plus ou moins délavés recouvrent de vastes surfaces sur les plateaux caillouteux du Bas-Rhône, particulièrement la Costière du Gard. Quelques placages moins étendus et moins caractéristiques jalonnent la Crau au S. des Alpilles. Au S. E. d'Aureilles, dans une carrière sise le long de la route de Mouriès à Eyguières, nous avons observé une petite couche de loess de $10 \mathrm{~cm}$, cryoturbée, qui reposait sur des formations constituées par un mélange de matériel fluvio-glaciaire alpin déposé par la Durance et de coulées de solifluction locales formées de calcaire jurassique. Elle était recouverte par d'autres coulées de solifluction identiques, mais sans aucun matériel alpin, qui recoupaient le premier ensemble de couches. On peut donc affirmer que le loess d'Aureilles, cryoturbé et recouvert par des pierrailles périglaciaires, est antérieur à la dernière période froide.

On retrouve des loess étendus mais de facciès parfois local sur les affleurements des bassins molassiques d'Uzès et d'Alès. Quelques taches exigües apparaissent aussi çà et là sur les plateaux calcaires de la Garrigue de Nîmes, comme l'a signalé Marcelin (4).

Il ne nous a pas été possible de nous livrer à la cartographie détaillée des lambeaux de loess de cette région mais nous avons pu néanmoins remarquer que la formation avait une importance croissante en direction du Rhône. Du côté de l'Est, affleurements de la Crau au S. des Alpilles sont réduits Au N., au pied de la Montagnette (notamment au S. de Barbentane), apparaissent des placages plus étendus, mais souvent à faciès très calcaire, peu caractéristique, que la feuille Avignon a cartographiés sous la rubrique A. Au N. de Pont St. Esprit, la terrasse fluvio-glaciaire suivie par la voie ferrée en montre une épaisseur de plus de $2 \mathrm{~m}$, entaillée par la tranchée de la route. Suen (19) en signale d'importants gisements sur la basse Céze, la basse Ardèche, Marcelin dans la Valbonne. En s'écartant du Rhône vers le S.O., les loess se raréfient: les environs de Montpellier n'en montrent que des traces peu épaisses sur les cailloutis pliocènes. Vers Béziers et Pézenas nous n'en avons plus relevé d'affleurements indubitables. Le lambeau caractéristique situé le plus loin vers le N.O. que nous ayions pu identifier se trouve le long des pentes S. E. du petit Bassin d'Aniane oú il recouvre des coulées de solifluction venant s'intercaler dans les alluvions de l'Hérault.

$2^{\circ}$ Les cailloux é olisés. Le Languedoc oriental présente quelques uns des plus beaux cailloux éolisés qu'il nous ait été donné de voir. Aux environs de Rochefort, de Tavel, de Pujaut, des formations alluviales anciennes riches en quarzites et proches de dépôts de sables Pliocènes montrent, à même la surface du sol, des multitudes de quarzites à facettes. Sur certains d'entre eux, l'ablation a pu atteindre 6 ou $7 \mathrm{~mm}$ d'épaisseur par endroits. Marcellin en a donné une magnifique photographie (4).

Les quartzites éolisés atteignent jusqu'à 15 et $20 \mathrm{~cm}$. de long, la majorité ayant autour de $10 \mathrm{~cm}$. Leur poids dépasse généralement $400 \mathrm{gr}$. et avoisine souvent $1 \mathrm{~kg}$. De tels galets soùt trop sourds pour avoir pu être retournés par le vent. Or la quasi-totalité d'entre eux sont éolisés sur toutes leurs faces. Ils ont donc dû être retournés de temps à autre au cours même de la période pendant laquelle ils s'éolisaient. Nous pensons, avec A. CaIlleux (7), que la meilleure explication de ce fait est d'admettre un retournement par le gel, par exemple sous l'effet des pipkrakes. Il y a donc tout lieu de penser, à la suite de MArcelin, que l'essentiel de ces actions éoliennes est fossile et d'âge périglaciaire ce qui 
n'empêche pas la continuation de ces phénomènes à l'heure actuelle favorisés en partie par le défrichement agricole. Mais ils sont très atténués.

Tout comme les loess, les cailloux éolisés abondent particulièrement sur le bord O. du Bas-Rhône. Des recherches, sommaires il est vrai, ne nous ont pas permis d'en trouver dans la Crau ni aux environs de Montpellier et de Béziers alors qu'ils abondent dans la Costière, le long du Vistre et dans la dépression de l'Etang de Pujaut.

$3^{\circ}$ Les sables é oliens. Leur recherche exige de nombreuses prises d'échantillons et de longs examens au laboratoire qu'il ne nous a pas été pos sible de faire sur la large échelle souhaitée. Nous nous contenterons donc de signaler quelques faits, espérant attirer l'attention sur eux.

Des sables éoliens sont visibles dans la coupe de Collias (couche Ic). On en trouve également de nombreuses nappes en Camargue, sur les anciens cordons littoraux ou levées du Rhône, mais, dans cette dernière région, leur âge est fort incertain et ils continuent souvent à se déplacer de nos jours. En Costière, notamment aux environs de Générac, quelques échantillons de sables alluviaux pris sous la terre végétale nous ont montré un fort pourcentage de grains éolisés.

Enfin, aux abords de Rochefort, notamment entre ce village et Tavel, les sables pliocènes sont remaniés en petites dunes qui viennent recouvrir des formations à quartzites plus récentes. Cette couverture éolienne est fixée et recouverte d'un sol, ce qui prouve qu'elle est fossile. Aux environs, à la surface des formations caillouteuses, s'observent quelques pavages éoliens, dus à la concentration des éléments grossiers par déflation.

Comme les loess et les cailloux éolisés, ces phénomènes se concentrent sur le bord Ouest de la dépression du Bas-Rhône.

Il ne nous a pas été possible d'utiliser ces faits pour reconstituer la direction des vents lors des périodes froides quaternaires. Mais la répartition même de ces dépôts semble indiquer que le relief a joué, comme aujourd'hui, un rôle prédominant et que les courants atmosphériques les plus violents étaient de direction méridienne. Ainsi s'expliquerait aisément l'importance exceptionnelle des phénomènes éoliens de part et d'autre du Bas-Rhône.

b) Les phénomènes cryopédologiques.

Ainsi que l'a déjà signalé MARcelin, les formations périglaciaires non éoliennes du Languedoc Oriental appartiennent surtout aux dépôts de gélivation et de solifluction.

1.) Les phénomènes de gélivation. Ils ont, comme d'habitude, une importance variable suivant les roches.

Dans les formations alluviales, Marcelin (13) a signalé, en Costière, la fréquence des galets éclatés, aux cassures anguleuses, emballés dans les restes d'argiles d'altération. Il s'agit là d'une formation de gélivation typique développée au détriment d'alluvions, comparable au »bief à silex « de Picardie, de Thiérache ou de Normandie. Nous avons trouvé également, particulièrement dans la Crau, de nombreux galets éclatés par le gel, débités en lamelles. Souvent ce matériel a été ensuite consolidé en conglomérat et le ciment ressoude les éléments éclatés ce qui montre que la gélivation n'est pas récente.

Dans les Cévennes, notamment aux environs de St. Laurent le Minier et du Vigan, les schistes montrent également de nombreuses traces de gélivation. Les pentes de $30-40^{\circ}$ sont souvent recouvertes d'une couche de $0,5-1 \mathrm{~m}$. de débris aplatis, généralement longs de $10-20 \mathrm{~cm}$. Il ne s'agit pas de formations actuelles car elles sont recouvertes d'une mince pellicule de terre végétale et fixées 
par la végétation. Bien que la gélivation puisse se continuer de nos jours là oú la roche pointe à la surface du sol, la plus grande partie de ces débris est d'origine périglaciaire.

Mais c'est dans les régions calcaires que l'action de la gélivation est la plus évidente, particulièrement sur les Garrigues et les Causses. La roche en place est recouverte d'un tapis de pierraille qui continue de se former actuellement sous l'effet de l'éclatement par le gel lors des températures rigoureuses d'hiver (jusqu'à $-12^{\circ}$ en $1950-51$, d'après Marcelin), d'autant plus que, contrairement à ce qui se passe dans la France Atlantique, la roche n'est pas protégée par un sol épais et affleure souvent entre les buissons (en grande partie par suite des dégradations dues au pâturage). Mais ce n'est qu'un médiocre prolongement de phénomènes passés. La superposition de lagélivation actuelle à la gélivation quaternaire est particulièrement nette dans le petit vallon par lequel la route de St. Guilhem le Désert au Causse de la Selle s'échappe de la gorge de l'Hérault. Les versants en sont tapissés d'éboulis. Les débris quaternaires sont fixés par la végétation et leur surface, légèrement décalcifiée, commence de se colorer en jaune par suite de la formation d'argile. Les éléments sont plus petits (ils dépassent rarement $10 \mathrm{~cm}$. de long) et plus aplatis que ceux des éboulis actuels. Ils sont aussi mieux calibrés et légèrement ordonnés, la position à plat étant prédominante. Enfin leur pente est plus faible: $29-30^{\circ}$ contre $33-34^{\circ}$. Les éboulis actuels sont blancs, mal triés (blocs de $50 \mathrm{~cm}$. ou même $1 \mathrm{~m}$.), moins riches en granules et moins régulièrement disposés. Des têtes de roche en place leur correspondent toujours en haut du versant. La moindre pente des éboulis anciens semblerait indiquer une légère intervention de la cryoturbation dạs leur mise en place, comme, par exemple, une action des pipkrakes. Elle est en effet inférieure à la pente des éboulis de pure gravité, du type des éboulis actuels Cette différence de pente permet à ces derniers de raviner légèrement les éboulis périglaciaires.

L'intensite de la gélivation est étroitement liée au faciès des calcaires (14). Elle est maxima dans les calcaires marneux, comme le Barrémien des Alpilles car de minces lits de marne, épais de $3-5 \mathrm{~cm}$., intercalés entre des bancs de calcaire marneux de $30-40 \mathrm{~cm}$., gonflent sous l'effet du gel et permettent la dislocation de la roche. Toute la partie occidentale de la voûte anticlinale des Alpilles est fortement affectée par la gélivation et noụs y avons observé de magnifiques éboulis ordonnés (pente $20^{\circ}$, éléments les plus gros $4-6 \mathrm{~cm}$., aplatissement de 3,2 ), maintenant recouverts par une végétation forestière. La Montagnette, le bord Est de la dépression de l'Etang de Pujaut, les environs de Tavel et de Rémoulins montrent, dans le même étage et le même faciès, des accumulations considérables de produits identiques qui forment de puissants glacis au pied des versants et une véritable terrasse alluviale au bord S.O. de l'étang de Pujaut (aplatissement 3,6). Il s'agit d'un matériel de gélivation typique, semblable à celui que donnent les calcaires marneux du Bartonien des environs de Paris ou du Portlandien du Barrois.

Les calcaires de l'Hauterivien sont moins gélifs et donnent des produits moins aplatis (aux environs de 3 ) et moins bien calibrés (éléments allant des granules à $20 \mathrm{~cm}$. env.). Les produits de ce genre abondent au pied de l'escarpement qui borde la Garrigue de Nîmes vers le S.E., où ils ont été signalés par MARCELIN (4).

L'Urgonien présente un faciès plus résistant, dans lequel certains bancs seulement sont gélifs, d'où le développement de formes différentielles, notamment d'abris sous roches (voir Marcelin, 4). Les calcaires dolomitiques, enfin, qui 
apparaissent fréquemment sous la forme d'accidents dans les séries Jurassiques des Causses, sont immunisés contre la gelée et n'ont pas été affectés par les actions périglaciaires. Il en est de même de la molasse coquillière des Baux, en Provence.

$2^{\circ}$ Phénomènes de solifluction et de cryoturbation. Leur développement est moindre que celui des phénomènes de gélivation.

Malgré l'examen de nombreuses coupes, nous n'avons pu trouver aucune fente en coin, aucun sol polygonal ni quoi que ce soit que l'on puisse interpréter comme tel. Les cryoturbations les plus poussées qu'il nous ait été donné de relever sont le sol à poches (Brodelboden) de Collias, qui s'est développé dans des conditions lithologiques exceptionnellement favorables, dans une formation présentant la granulométrie optima (maximum granulométrique $20-50$ microns). Au coin S.E. de l'Etang de Pujaut, des débris de calcaire anguleux consolidés en brèche et formant une coulée de solifluction, ravinent des formations plus fines en formant des figures très contournées, esquissant des plications complexes (Würgeboden). Enfin, au N. de Pont St. Esprit, la coupe de la terrasse du chemin de fer, dans le talus de la route, nous a montré de jolies injections de matériel alluvial pénétrant en pincée de $0,5-0,6 \mathrm{~m}$. dans la base du loess sus-jacent. Tels sont les seuls phénomènes de cryoturbation que nous ayions pu observer malgré une recherche attentive. Marcelin est arrivé aux mêmes résultats: il ne signale que des poches ravinantes (coupes de coulées de solifluction) et quelques esquisses de guirlandes avec rebroussements (creux du Chaffre, près Calvisson, in $\mathrm{n}^{\circ} 4$, p. 111).

Par contre, les phénomènes de solifluction sont importants, massifs pourrait-on dire. Ils ont étalé au pied des versants calcaires des glacis considérables de débris, en pente faible, qu'a décrits Marcelin. La granulométrie de ces formations rend leur solifluction impossible sous climat tempéré: ce sont des pierrailles à matrice de granules et de poudre, sans argile. Au surplus, elles sont fixées depuis longtemps et des sols les couronnent. A la différence de ce qui passe dans la France du Nord, elle sont fréquemment consolidées en brèche par un ciment de calcaire. Les pentes les plus faibles que nous leur ayions observées sont de $2-3^{\circ}$. Etant donné leur importance primordiale sur le modelé, nous en reparlerons plus en détail au paragraphe suivant.

De ces divers faciès des phénomènes cryopédologiques nous pouvons essayer de tirer quelques conclusions concernant les climats froids du Quaternaire dans la région du Bas-Rhône. Les phénomènes liés au froid le plus intense, comme les fentes en coin, les sols polygonaux de grand diamètre, les injections puissantes, les plissotements marqués des couches manquent totalement ou bien sont rares et peu développés. Le climat ne fut donc pas t r ès rude, plus clément semble t'il que sur la côte atlantique à la même latitude, car Balland, BASTin et Cailleux, puis Guillien ont observé ces phénomènes en Charente (15). Il ne semble pas qu'il y ait aucune preuve d'un sol gelé permanent (merzlota). Toutefois le gel pénétrait chaque hiver profondément et était suffisamment intense pour éclater finement les roches gélives. La couche gelée devait être également suffisamment épaisse pour permettre, au printemps, une solifluction intense et prolongée de débris incapables de solifluer sous un climat tempéré, sans un sol gelé sous-jacent. Nous inclinerions donc à admettre que la région du Bas-Rhône a connu, au Quaternaire, à plusieurs reprises, un climat de toundra atténué, du genre de celui qui règne non loin de la limite septentrionale des forêts. $\mathrm{Au}$ même moment, le littoral atlantique connaissait des conditions plus sèvères, 
comme le montrent les découvertes de Guilcher. Il est probable que ces différences régionales sont liées à la proximité de la Méditerrannée, probablement presque coupée de l'Atlantique par suite de l'abaissement du niveau marin lié à la glaciation, et qui, de ce fait, devait être moins froide. Quoiqu'il en soit, il n'est plus possible d'admettre dans cette région un climat tempéré, comme on le faisait encore récemment (1).

Une grande partie des notions de morphologie climatique qui permettent seules de comprendre le relief de la France Septentrionale doit donc être étendue au Languedoc.

\section{L'Influence Morphologique des Phénomènes Périglaciaires}

L'influence des actions périglaciaires fossiles sur le modelé actuel dépend d'une part de leur intensité propre et de l'autre de l'intensité des processus postglaciaires. Là où les actions périglaciaires fossiles ont été réduites et où les processus actuels sont actifs, l'influence des premières a pu être complètement effacée et leur rôle morphologique devient négligeable. Par contre, lorsque le froid quaternaire a permis le développement d'un modelé original et vigoureux et que les actions postérieures n'ont guère eu d'importance, le modelé actuel est presque entièrement un modelé périglaciaire fossile conservé.

Un exemple d'une médiocre influence morphogénétique des phénomènes périglaciaires fossiles nous est offert dans le Languedoc par les régions argileuses ou molassiques. Ce n'est pas que les climats froids ne les aient pas affectées: la molasse ou l'argile ne jouit d'aucune immunité vis à vis de la cryoturation. Cette dernière a provoqué dans ces formations une importante solifluction qui a contribué à la progression de l'ablation. Mais les formes qui se sont développées sous l'effet des climats froids n'ont pas persisté jusqu'à nous. Par suite, en grande partie, de la mise en culture, la molasse et l'argile, formations meubles, sont la proie de l'érosion des sols. Cette dernière est suffisamment active pour développer toute une micro-topographie de ruissellement, caractérisée par des ravins et ravineaux rapprochés qui dissèquent les anciens versants amples, aux formes plus douces et plus régulières, qui s'étaient établis sous l'effet de la solifluction. Quelques fois, comme sur la butte au N. de Collias, les anciens versants, antérieurs à la phase actuelle de ruissellement, persistent encore sous la forme des sommets de croupe qui séparent les ravineaux. D'autres fois le recoupement des versants les a fait totalement disparaitre, comme aux Fosses de Fournès.

Il n'en est pas de même des calcaires. Dans ces roches, en effet, l'érosion post- et interglaciaire est essentiellement chimique, et, de ce fait, fort lente. C'est ce que BIRot désigne par l'expression d' »immunité karstique«. Par contre, lors des périodes froides du Quaternaire, l'érosion mécanique était fort intense. Gélivation et solifluction se combinaient pour provoquer l'ablation de masses de matériaux considérables. Il en est résulté une intense érosion mécanique que n'a pu modifier la médiocre action postglaciaire, exactement comme en Lorraine ou en Champagne (10). L'essentiel des versants porte aujourd' hui encore l'empreinte périglaciaire. Naturellement cette dernière est plus ou moins profonde suivant la gélivité des roches. Dans les calcaires marneux, elle est à peu près exclusive. Dans les dolomies, au contraire, elle est nulle et ce sont des formes plus anciennes, pré-glaciaires, qui sont conservées.

Toutes les régions calcaires du Languedoc Oriental et de la Provence Occidentale sont caractérisées par l'opposition entre les plateaux ondulés, faiblement 
accidentés qui règnent sur de grandes surfaces et les gorges abruptes, les canyons aux parois raides où coulent les rivières (Gorges de l'Hérault, du Gard, de la Vis etc...) Seule la morphologie climatique, appuyée sur ce que nous venons de préciser quant aux paléoclimats quaternaires, est susceptible d'en founir une explication compléte. Im ne s'agit pas là d'une morphologie homogène, prétendue karstique, mais de formes d'âge varié, de genèse différente, juxtaposées par suite de l'évolution climatique.

Les gorges sont le domaine où se poursuit une érosion mécanique actuelle. Le vallon entre St. Guilhem du Désert et le Causse de la Salle nous en fournit une preuve qui n'est pas unique. Partout oú l'enfoncement des rivières est suffisamment actif, les versants calcalres évoluent en talus d'éboulis, le gel actuel jouant encore un rôle essentiel dans la fragmentation de la roche, bien que fort intérieur au gel quaternaire. Dans la gorge du Gard à travers l'anticlinal urgonien de la Garrigue de Nîmes, le sapement du fleuve favorise également les éboulements. Ici aussi les versants actuels sont des versants d'éboulis. Quelques bancs plus résistants donnent des petits gradins verticaux et des sections de versant d'une pente moyenne de $60-70^{\circ}$. Entre eux, les éboulis donnent des talus de 33 à $35^{\circ}$, un peu plus inclinés que les éboulis périglaciaires. A l'action de sapement des rivières qui provoque des éboulis de gravité se borne la morphologie superficielle postglaciaire.

Par elle, les interfluves ne sont pas touchés. Leur surface est ondulée par l'entaille de toute une série de vallons, aujourd'hui secs et qui n'ont pu se former sous le climat actuel. Leurs versants sont jonchés de pierrailles et, cependant, leur pente est bien inférieure à celle des talus d'éboulis. Pour le plateau Urgonien de la Garrigue de Nîmes, elle est le plus souvent de $10-20^{\circ}$. Seule une action du ruissellement pourrait expliquer semblable valeur. Or, dans ces roches perméables, il ne saurait en être question. Cette morpologie de vallons secs, aux versants relativement adoucis, toujours moins raides que ceux des canyons à écoulement fluviatile sont un legs des climats froids périglaciaires. Or c'est à eux que la garrigue doit l'essentiel de son relief.

La pente de leurs versants varie directement en fonction de la gélivité des roches: le plus souvent voisine de $20^{\circ}$ dans les calcaires urgoniens, elle s'abaisse à $10^{\circ}$ dans les calcaires marneux du Barrémien ou de l'Hauterivien.

Une morphologie légèrement différente caractérise le rebord des plateaux calcaires, par exemple le grand talus qui s'allonge tout au long de la Garrigue de Nîmes, le long du Vistre. Nombreux sont les vallons secs qui l'entaillent, semblables, comme les précédents, aux vallons en berceau de la Lorraine (10). Aucune trace d'eau courante, pas même de lit dessiné, ce qui montre bien qu'ils sont totalement inactifs de nos jours. Par contre leur fond est tapissé d'une épaisse nappe de pierraille de gélivation, apportée là par la solifluction, et qui empâte le pied des versants, formant un long glacis en pente douce $\left(4-5^{\circ}\right)$ raccordant la nappe du fond de vallon aux affleurements de roche en place, dont la pente est sensiblement plus forte. Entre les vallons, c'est le pied même du talus qui est bordé de ces glacis continus et réguliers, paysage caractéristique de la vallée du Vistre, comme l'a bien sougligné Marcelin. Entre Nîmes et Rémoulins le grand développement de ces vallons et de ces glacis s'explique par l'absence d'entaille récente. Une coupe proche de l'aérodrome de Nîmes, que nous a montré MARcelin, laisse voir les formations de débris de gélivation soliflués consolidés par un ciment calcaire presque jusqu'au niveau de la surface du sol. Cette consolidation est fossile et date probablement du dernier inter- 
glaciaire. Lors de la dernière période froide, les horizons supérieurs du sol dont elle représente la base ont disparu sous l'effet de la reprise de la solifluction. Or cette brèche se raccorde à peu près au fond du vallon, où elle n'est recouverte que de $1 \mathrm{~m}$. environ de débris. Il n'y a donc eu, dans cette région, aucun creusement sensible entre les deux dernières périodes froides de sorte que l'évolution commencée pendant la première a pu se poursuivre sans accident au cours de la seconde.

Une morphologie comparable s'étend au pied de l'escarpement Barrémien qui borde au S.E. la dépression de l'étang de Pujaut. La roche en place, inégalement gélive, est découpée en pinacles séparés par des couloirs ennoyés sous les débris de gélivation. Ces derniers se rejoignent plus bas pour former un glacis continu. Les rivières autochtones ont façonné ce matériel en grève calcaire, très aplatie et faiblement émoussée, aux éléments de forme subgéométrique, exactement semblable au matériel périglaciaire fluviatile que l'on rencontre si souvent, dans le Bassin de Paris (16).

Les mêmes pentes façonnées avant tout par la gélivation et la solifluction caractérisent la topographie des petits Causses (Larzac, Causse de la Selle). Des glacis de débris de gélivation adoucissent le bord de la plupart des dépressions karstiques. Une étude détaillée des rapports entre ces formations et la topographie karstique permettrait de dater cette dernière et montrerait probablement que l'essentiel du travail de dissolution est ancien.

Au S. de Montdardier, entre St. Martin et la Vacquerie, nous avons observé en de nombreux points une morphologie karstique fossilisée sous des dépôts de sables à galets de quartz, attribués au Pliocène par la carte géologique. Le bord des poljés est ennoyé sous des glacis de poduits de gélivation. Il faut donc tenir le plus grand compte des phénomènes périglaciaires quaternaires pour expliquer la morphologie des Causses. Partout où le calcaire est gélif, les formes de surface ont été effacées par l'action du froid: les lapiés ont disparu, les dollines les plus petites ont été complètement fossilisées, les plus grandes presque effacées *). Seules ont pu se maintenir les formes développées dans des faciès non gélifs, en particulier dans les formations dolomitisées. Ainsi s'expliquent les lapiès de Rogues, de la Cavalerie, de la Vacquerie et de probablement bien d'autres points. Il s'agit de formes anciennes, antérieures au dépôt de la nappe de galets de quartz à Rogues et à la Vacquerie (elles sont fossilisées par elle), qui se sont maintenues par suite de l'immunité que leur conférait le faciès vis à vis de la gélivation. Ce n'est pas à un karst actuel que nous avons à faire, mais à un vieux karst, superficiellement abîmé par les périodes froides quaternaires, et dont seules les parties souterraines ont pu se remettre à fonctionner sans difficulté lors du radoucissement du climat tandis que la surface est encore largement un paysage périglaciaire fossile. Ainsi s'explique l'apparente disparité d'évolution entre les formes souterraines et les formes superficielles. L'analogie est grande avec le Jura Souabe et les Alpes arientales (Voir BüDEL No. 21).

La châine des Alpilles montre un contraste saisissant, de morphologie calcaire. Toute la partie centrale de l'anticlinal, constituée par le Cretacé inférieur et le Jurassique supérieur, montre une intense dissection par un réseau serré de vallons secs, dont les versants ont des pentes modérées $\left(10-20^{\circ}\right)$. Surtout dans le Crétacé Inférieur, le relief est aéré, les vallons évasés. Les confluences de ravins sont extrêmement rapporchées: tous les $100 \mathrm{~m}$. en moyenne le long de

*) Cette idée nous a été suggérée par R. Dugrand, que nous remercions cordialement. A. Callleux a observé au Djurdjura (Algérie) des faits analogues (17). 
la route de St. Rémy aux Baux. On observe d'abondantes formations de gélivation, en particulier des éboulis ordonnés. Aucune morphologie karstique, si ce n'est que tous ces vallons sont secs. Tout le modelé est périglaciaire et les périodes froides du Quaternaire ont mis à rude épreuve le coeur de l'anticlinal. C'est qu'il est constitué de calcaires gélifs, souvent marneux (Grétacé Inférieur).

Par contre, une fois l'anticlinal franchi en direction du S., la région des Baux offre un relief chaotique, avec dépressions fermées (le Val d'Enfer), buttes résiduelles du type hum, aux parois verticales toutes taraudées d'énormes lapiés. Ici, aucun éboulis ordonné, aucune trace de solifluction. Le modelé est tout entier celui de l'érosion chimique. C'est que nous nous trouvons dans da molasse aquitanienne, constituée par un calcaire coquillier tendre, non gélif, utilisé comme pierre de taille. Les formes fossiles préglaciaires sont intégralement conservées.

La même influence morphoclimatique s'est fait sentir sur le travail des rivières et la morphologie des fonds de vallées. Il est très vraisemblable qu'ici comme dans tout le Bassin de Paris, la plupart des terrasses soient climatiques (18). Le remblaiement du Bassin d'Aniane est formé de coulées de produits de gélivation venant se fondre dans les apports longitudinaux ce qui permet de le dater d'une période froide. Mais plus significatif est un petit vallon descendant du flanc E. de la Gardiole, près de Mireval et dont le matériel est entaillé par une carrière le long de la route de Frontignan à Montpellier. Il a édifié un cône, formé uniquement de matériel calcaire local, très peu usé, mais assez aplati. Large de $200 \mathrm{~m}$. à sa base, il atteint $7 \mathrm{~m}$. de puissance et repose sur le calcaire fortement altéré par dissolution et encore bourré d'argile rouge dans les diaclases élargies. Le calcaire était entaillé par un petit ravin profond et étroit, qui a été entièrement fossilisé par le cône. De quand date ce ravin? Probablement de la période où les couches de l'ensemble II se ont altérée à Collias: en effet, la surface du calcaire du ravin fossilisé porte l'altération rouge tandis qu'au dessus du remblaiement, la même roche, fortement gélivée, est intacte. Les formations du cône se raccordent aux pierrailles de gélivation qui jonchent cette roche en place: elles sont postérieures à l'altération rouge. Mais ce n'est pas tout: elle-mêmes plongent vers la plaine littorale toute proche et passent sous les formations argilo-vaseuses qui bordent l'étang de Vic. Elles sont donc antérieures à la transgression flandrienne D'ailleurs ce cône n'est plus fonctionnel: il est recouvert d'un sol mince, brun, qui semble postglaciaire et aucun écoulement ne se produit plus dans le talweg. On exploite la carrière sans se soucier de ce dernier.

La formation du cône s'est donc placée lors des deux récentes phases froides correspondant au depôt des loess Ib et Id de Collias, c'est-à-dire lorsque le niveau de la mer toute proche était déprimé par la glaciation. Au contraire, lors de la transgression flandrienne, la remontée du niveau marin a coîncidé avec un arrêt de l'accumulation. Indépendante des oscillations eustatiques, l'évolution de ce vallon est essentiellement commandée par les fluctuations climatiques, comme celle de la plupart des rivières françaises.

L'existence de périodes froides au Quaternaire dans le Midi Méditerranéen, prouvée par l'existence de dépôts typiques, attestée par une faune froide, notamment le renne au Mas d'Azil et des pingouins fossiles en Italie (d'après M. Gignoux), est donc d'une importance capitale pour la géomorphologie. Les oscillations climatiques ont en effet engendré le développement de générations de formes de caractère très différent, qui ont persisté plus ou moins jusqu'à nous. Tandis que le modelé des argiles ou dela molasse répond largement aux 
conditions climatiques actuelles, celui des calcaires, sauf le long des canyons, est entièrement fossile. Des topographies souterraines tertiaires ou quaternaires anciennes s'y combinent avec un modelé périglaciaire récent pour constituer un assemblage étrange dont on a voulu faire un type de modelé karstique.

La meilleure connaissance de ces divers paléoclimats et des formes caractéristiques qu'ils ont engendrées nous aidera également à mieux préciser les étapes de l'évolution du relief en permettant de dater telle ou telle forme grâce aux sols climatiques qui la recouvrent. Puissent de telles recherches, appuyées sur l'étude minutieuse des formations superficielles et des processus de l'érosion, se multipier et nous permettre de mieux comprendre les topographies qui se présentent à nos yeux.

\section{Ré férences bibliographiques}

1 BüDEL (J): Die räumliche und zeitliche Gliederung des Eiszeitklimas. - Die Naturwissenschaften, 36, p. 105-112 et 133-39. 1949.

2 Guilcher (A): Sur les vallons suspendues des environs de Lisbonne et leurs analogies avec ceux du littoral breton. - C. R. Ac. Sc., 228, p. 1512-4. 1949.

3 BAeckeroot $(\mathrm{G})$ : Observations préliminaires sur des formes quaternaines d'origine climatique dans la Montagne Noire Occidentale. - Bull. A. G. F. 1951, p. 34-7.

4 Marcellin (P): Phénomènes du vent et du froid au Quaternaire Supérieur dans la région Nîmoise. - Bull. Soc. Languedoc. de Géogr. 2, p. 85-122. 1950.

5 Marcellin (P): Observations sur des terres et des sols en région méditerranéenne, I Terres et sols en Costière. Nîmes 1947.

6 Cailleux (A): Interprétation climatique des éolisations pliocènes et quaternaires en France. - C. R. Somm. S. G. F. 1951, p. 44-6.

7 Doeglas (D. J.): Loess, an eolian product. - Sedimentary Petrology, 1949, p. 112-7.

Callleux (A): Les actions éoliennes périglaciaires en Europe, Thèse Sc. - Mém. S. G. F. 176 p. Paris 1942.

8 Poser (H): Zur Rekonstruktion der Spätglazialen Luftdruckverhältnisse in Mittelund Westeuropa auf Grund der vorzeitlichen Binnendunnen. - Erdkunde 4, p. 81-88. 1950.

Poser (H) \& Tricart (J): Les terrasses de l'Huisne en amont du Mans. - B. S. G. F., 1950, p. 381-91.

Tricart (J): Dépôts Würmiens et post Würmiens du cône de déjections de la Bruche, près de Strasbourg. - C. R. Somm. S. G. F., 1950, p. 138-9.

9 Guilcher (A) \& Calleux (A): Reliefs et formations quaternaires du Centre-Est des Pays-Bas.Rev. de Génomorphologie Dynamique 1, p. 128-42. 1950.

10 Tricart (J): La Partie Orientale du Bassin de Paris, Thèse Lettres, Paris 1948, Ed. S. E. D. E. S., Tome II, 1952.

11 Tricart (J): Les formations quaternaires de la feuille Vouziers au 1/50.000. - Bull. Serv. Carte Geol. de France, 1950, p. 209-24.

12 Tricart (J): Compte rendu de l'excursion de Valence, du 18 au 24 Février 1950. Bull. Fac. Lettres Strasbourg 1950, p. 384-96.

13 Marcelin (P): Contribution à l'étude de la Garrigue Nîmoise. - Etudes Rhodaniennes 2, 1926, p. 83.

14 Tricart $(J)$ : Le modelé des régions froides. $1^{\circ}$ partie: le modelé périglaciaire. 267 p. C. D. U., Paris 1950.

15 Balland (R) \& Cailleux (A): Etude morphoscopique de quelques sables de la région bordelaise. - C. R. Somm. S. G. F. p. 58-7, 1946.

Bastin \& Cailleux (A): Action du vent et du gel au quaternaire dans la région bordelaise. - B.S. G. F., $5^{\circ}$ ser., 11, p. 259-66.

Carlleux (A): Les formations superficielles de la feuille Saintes au $1 / 80.0000^{\circ}$. B. Serv. Carte Géol. Fr., p. 131-7, 1948.

Guillien (Y): Pour la chronologie de la période froide: les données charentaises. Sess. extraord. Soc. belges de Géol. Bruxelles 1946, p. 347-61.

16 Tricart (J) \& Schaeffer (R): L'indice d'émoussé des galets, moyen d'étude des systèmes d'erosion. - Rev. de Géomorph. Dynamique 1, p. 151-179. 1950.

17 Barbier (A) \& Cailleux (A): Glaciaire et périglaciaire dans le Djurdjura Occidendal. - C. R. Ac. Sc. 231, p. 365-6. 1950.

18 Tricart (J): Premiers resultats d'une synchronique des terrasses du Bassin de la Seine. - C. R. Somm. S. G. F. p. 69-71, 1946. 
19 Suen (T. Y.): Le loess de la vallée, du Rhône. - Thèse Lettres (Université), Lyon 1934.

20 Viret: Bull. Soc. Linnéenne de Lyon 18, p. 20-24. 1949.

Bourdier (F): C. R. Ac. Sc. 215, p. 422.1942.

21 Büdel (J): Fossiler Tropenkarst in der Schwäbischen Alb und den Ostalpen. Erdkunde 5, 1951.

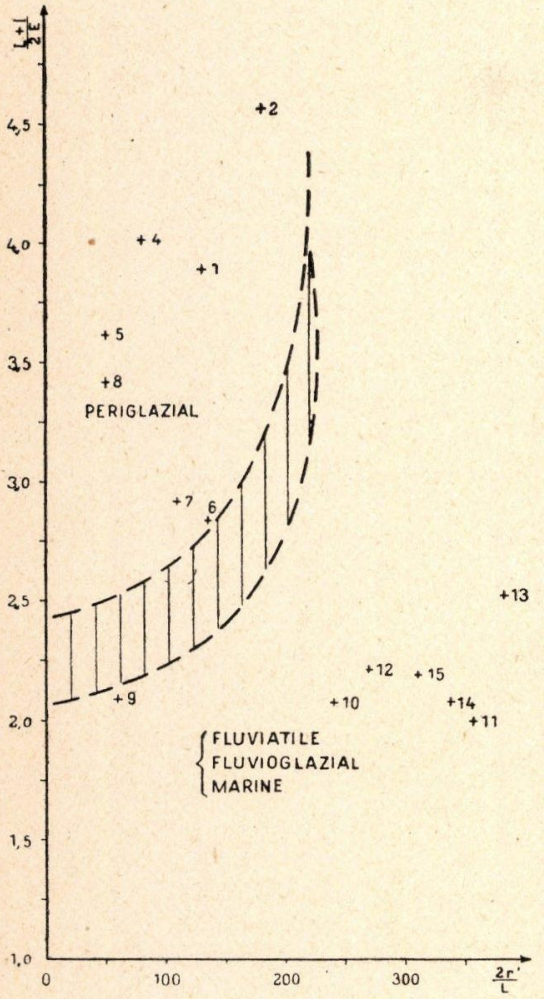

FIGURE 3: Caractéristiques morphométriques des formations périglaciaires grossières étudiées. En abscisses, indices d'émoussé (médianes de chaque lot), en ordonnées, indices d'aplatissement (medianes de chaque lot). Chaque lot est figuré sur le graphique par un croix accompagnée d'un chiffre renvoyant au tableau 1 . On peut constater que les caractéristiques morphométriques des galets périglaciaires sont bien différentes de celles des autres formations. Elles se répartissent sur une aire bien délimitée correspondant à des émoussés médiocres ou nuls et des aplatissements élevés ou moyens. Ces caractères conistituent un éventuel moyen de diagnose d'origine. On notera également que les formations périglaciaires du Bas Rhône se placent au bord de l'aire délimitée sur le graphique: leurs caractères sont moins nets que ceux dies formations de la France du Nord et indiquent une transition, un climat moins rude.

T A B L E U 1

Caractéristiques morphoscopiques des galets périglaciaires de la région du Bas Rhône et termes de comparaison.

Localité

Indice d'aplatissement

Indice d'émoussé
(1)
(2)
(3)

(4)

(5)

(6)

$1^{\circ}$ Formations périglaciaires de l'Est du Bassin de Paris

2 FALAISE (Ardennes)

3 CHEVIERES (Ardennes)
1 MARCQ (Ardennes)

$\begin{array}{llrll}3,87 & 37 \% & 50-150 & 0 \% & 130 \\ 4,55 & 13 \% & 100-150 & 1 \% & 180 \\ 5,3 & 19 \% & 200-250 & 2 \% & 195\end{array}$

$\begin{array}{llllll}5,3 & 19 \% & 200-250 & 2 \% & 195\end{array}$

$2^{\circ}$ Formations périglaciaires du Sillon Rhodanien

\section{OURCHES (Drôme)}

5 PUJAUT, éb. ordon.

6 PUJAUT, alluvions

7 AUREILLE (B. d. R.)

8 Les BAUX (B. d. R.) LEDENON (Gard)

$\begin{array}{rllllr}7 \% & 2,75-4 & 31 \% & 3,6 & 61 \% & 50-100 \\ 20 \% & 1,75-2,75 & 28 \% & 2,8 & 22 \% & 100-150 \\ 19 \% & 1,75-2,5 & 37 \% & 2,9 & 43 \% & 50-150 \\ 37 \% & 2,25-3 & 39 \% & 3,4 & & \\ 11 \% & 2,5-3,5 & 27 \% & 2,87 & & \end{array}$

$0 \% \quad 80$

40

$0 \%$

$0 \% 10$

30

$3^{\circ}$ Formations non périglaciaires, à titre de comparaison

9 JOUX La Ville

10 VOGÜE (Andèche)

11 FRAUENHOFEN

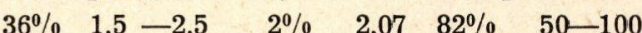

$\begin{array}{llllll}49 \% & 1,5-1,75 & 3 \% & 2,08 & 10 \% & 200-250\end{array}$ 
Localité

12 WERTHENSTEIN

13 WIBLINGEN

14 OUJDA (O. Isly)

15 SETE (Plage)

(1) Proportion des galets < 2. (2) Maximum. (3) Proportion des galets > 3,5. (4) Médiane.

(5) Proportion des galets < 100. (6) Maximum. (7) Proportion des galets > 500. (8) Médiane.

Renseignements concernant les formations de comparaison:

Joux la Ville: dissolution sur place actuelle dans un sol de rendzine.

Voogüé: fluviatile actuel, dans le lit de l'Ardèche.

Frauenhoffen: fluviatile tortonien de Bavière, aucune influence périglaciaire pasible. Werthenstein: fluviatile actuel de la Kleine Emme (Suisse).

Wiblingen: terrasse fluvio-glaciaire du Danube, près d'Ulm. Quelques éléments de provenance périglaciaire.

Oujda: fluviatile actuel de l'Oued Isly. Noter la ressemblance avec Frauenhoffen.

Sète: usure marine actuelle au pied d'une petite falaise, sans transport.

\section{$\mathrm{Z} u$ is a m m e $\mathrm{n}$ a s s u $\mathrm{ng}$}

Die bis in jüngste Zeit vertretene Ansicht, daß die französische Mittelmeerregion im Pleistozän nicht mehr unter den Einflüssen eines periglazialen Klimas gelegen habe (1), bedarf nach den Beobachtungen von MARCELIN (3) und nach den Feststellungen in diesem Aufsatz einer Revision. Auch in diesem Gebiet gibt es einen mannigfaltigen periglazialen Formenschatz und verbreitet periglaziale Ablagerungen (Fig. 1): Löße, Flugsande und äolische Steinpflaster (mit allseitig geschliffenen Steinen darin, deren größte und schwerste während der Politur nicht durch Wind, sondern nur durch Frostschub gedreht sein können), Solifluktions- und Kryoturbationshorizonte (Würgeboden) und periglaziale Schutthalden. Morphometrische Schotteranalysen, die Abplattung wie die Zurundung betreffend, erhärten den periglazialen Charakter dieser Bildungen (Tableau 1), deren Verbreitung bis ans Mittelmeergestade reicht. Die genaue Datierung der einzelnen Erscheinungen ist noch nicht möglich gewesen; sie gehören aber zumindest bei Collias verschiedenen Kaltzeiten an. Hier zeigt ein großer Aufschluß drei verschiedenaltrige Löße in Wechsellagerung mit Solifluktions- und Kryoturbationshorizonten und alle diese Horizonte mit unterschiedlichen Merkmalen nachträglicher chemischer Verwitterung. Er dokumentiert die Aufeinanderfolge folgender Klimaperioden: warm-trockene Klimaperiode, ältere Kaltklima-Periode, warm-trockene Periode, kalte Periode, gemäßigte Periode, kalte Periode, Klima der Gegenwart. Bemerkenswert ist, daß trotz Suchens keine fossilen Eis- oder Lehmkeile und auch keine anderen Zeugen einstigen Dauerfrostbodens gefunden wurden. Ihr offenbares Fehlen gestattet den Schluß, daß das durch die übrigen Formen und Ablagerungen belegte Periglazialklima nicht ebenso streng und rauh war wie das gleichzeitige Klima in den nöndlichen und westlichen Teilen Frankreichs, wo fossile Eiskeile bis an die Atlantikküste hin beobachtet wurden. Das französische Mittelmeergebiet wird also keinen Dauerfrostboden, sondern nur jahreszeitliche, bestenfalls tiefgründige Gefrornis gehabt haben.

Der Nachweis periglazialer Klimaeinflüsse auf dieses Gebiet eröffnet neue Aspekte auch für die Morphologie der südfranzösischen Kalkgebirge. In Abschnitt III sind zahlreiche Einzelbeobachtungen zusammengestellt worden, wonach manche Züge der großen kañonartigen Täler sowohl hinsichtlich ihrer Hanggliederung wie hinsichtlich ihrer Terrassen nur aus dem Wechsel der verschiedenen Klimate, also auch unter Mitwirkung des Periglazialklimas verstanden werden können. Eindeutig periglaziale Veränderungen weisen die Karsterscheinungen auf, deren Anlage somit weit älter ist, als bisher angenommen wurde. Vollends periglazialer Entstehung sind die zahlreichen, heute trockenen Tälchen der Hochflächen („Dellen“). Bedarf die Kalklandschaft im einzelnen auch noch weiterer Untersuchung, so darf doch schon gesagt werden, daß ihre Formen größtenteils Vorzeitformen aus verschiedenen Klimaperioden sind, und daß ihre jetztzeitlichen Bildungen sich nahezu ganz auf den Sohlenbereich der großen Täler beschränken. Durch die auffallend gute Erhaltung eines verschiedenaltrigen fossilen Formenschatzes zeichnet sich die Kalklandschaft aus vor den Räumen toniger $\mathrm{Ab}-$ lagerungen und der Molasse, die unter der Wirkung des heutigen Klimas noch am stärksten verändert werden. 